\title{
An algorithm for the Decentralized Market Coupling Problem
}

\author{
P. Basagoiti ${ }^{1}$, J. J. González ${ }^{2}$ and M. Álvarez ${ }^{3 *}$ \\ ${ }^{1}$ OMEL, Spain \\ Alfonso XII,6 \\ 28014 Madrid, Spain \\ Phone +34 916598933 \\ E-mail: pbasagoiti@omel.es \\ ${ }^{2}$ OMEL, Spain \\ Alfonso XII,6 \\ 28014 Madrid, Spain \\ Phone +34 916598926 \\ E-mail: jjgonzalez@omel.es \\ ${ }^{3}$ ETSI Industriales, UPM, Spain \\ José Gutiérrez Abascal, 2 \\ 28016 Madrid, Spain \\ Phone +34 913363205 \\ E-mail: malvarez@etsii.upm.es
}

\begin{abstract}
Keywords---Market Coupling, Block Bids, Lagrangian Relaxation.

Abstract--In this communication a conceptual algorithm is proposed for the market coupling process in the presence of block orders. The basic idea is to use the Lagrangian relaxation to get a decomposition of the problem that emulates the necessary decentralization process. The feasibility of this procedure is analysed using a simplified model of the problem. In particular, the solution obtained in this decentralized way is contrasted with the numerically exact solution of the problem by evaluating the duality gap. The examples presented suggest that in this way reasonably approximate solutions can be obtained, to improve those obtained through empirical procedures used by the market operators.
\end{abstract}

\section{INTRODUCTION}

Probably the most widely accepted methodology for the integration of the European Electricity Markets is the so-called Flow based Market Coupling. Jointly proposed by ETSO and EuroPEX, the basic accepted assumption is that the EEM can be operated as a series of single-price zones that can be linked by coupling their markets, while respecting the capacity of the interconnections.

An essential component of flow based market coupling is the implementation of a working procedure for coupling the electricity markets at the day-ahead stage in a decentralized

\footnotetext{
* Financial support by OMEL and partially by Project MTM2005-08897 of Ministerio de Educación y Ciencia of Spain.
}

manner, so that each market maintains its own rules of operation. With market coupling, the daily cross-border transmission capacity between various areas is not explicitly auctioned among the market parties, but is implicitly made available via energy transactions on the power exchanges on either side of the border. In this way, by allowing that the cheapest generation of the system can meet the demand in any market while maintaining their autonomy of operation, the total economic surplus will be maximized and a global price reduction is usually obtained.

The coupling of two electricity markets when working in a single period (hour) basis is conceptually simple. It is even possible to solve the problem graphically through the so-called export-import curves, which provide the modification of prices in each market as a function of the energy exchanged with the neighbour market. To create these curves, each market determines the local clearing price and a curve that indicates the price dependency as a function of the changing import/export volume. Using these curves and the known transport capacity a coupling is applied that, without congestions, results in a single price. This scheme can be extended to the case where there are more than two markets.

However, the situation changes drastically when the market rules permit the presentation of complex bids, typically in the form of indivisible multi-period energy blocks. Even in the case of a single market, this is a complex optimization problem, and in the European markets, iterative heuristic procedures are usually applied to obtain the clearing prices. The extension of these procedures to several coupling markets is much more problematic, and it is necessary to establish simplifying and empiric criteria to reach a solution. 
The problem of a matching algorithm dealing with complex bids may be formulated as a problem of mixed programming and, at least theoretically, it can be numerically solved using appropriate software. From this perspective, in this communication, a conceptual algorithm for market coupling between electricity markets in the presence of complex bids is proposed. The basic idea is to use the Lagrangian relaxation to get a decomposition of the problem that emulates the necessary decentralization process. The feasibility of this procedure is analysed using a simplified model of the problem. In particular, the solution obtained in this decentralized way is contrasted with the numerically exact solution of the problem by evaluating the duality gap. The examples presented suggest that in this way approximate solutions can be reasonably obtained, improving those obtained through empirical procedures currently used by market operators.

\section{MARKET COUPLING}

\section{A. Auction with complex bids}

In European electricity markets, the day ahead price is determined mainly by the matching of demand and generation bids, defining the hourly prices by the intersection points of inverse supply and demand curves. This procedure is justified because it ensures the maximum surplus of the market. To facilitate that the market participants could adapt their offers to its cost structures, it is common practice to allow participants in the markets to offer complex bids with fixed and indivisible volumes extended in several hours and considered firm only if hourly prices results in a minimum average price. The presence of this type of block orders complicates the auction process and all European electricity markets have established more or less empirical procedures to obtain the clearing prices. Essentially. these procedures first make the hourly auction by incorporating complex bids as if they were simple, checking subsequently if block volumes are included and if the averaging prices are compatible with the block price limitations. After removing those block orders that do not comply with their imposed conditions, the process is repeated until an acceptable situation is obtained. This process usually ends with the undesirable result of paradoxically rejected block orders, because of their prices are lower than the market price.

The problem of multi-period auction bids in the presence of complex blocks can be posed as a minimization problem. Our formulation is developed under assumptions that set aside many important aspects of the market reality. It is intended only to highlight the computational difficulties inherent to this problem, even in a very simplified version, complicating its effective numerical solution.

The supply of generation is modelled as follows. First, we suppose a supply of continuous generation with variable increasing price, whose volume in each hour can be set free and unlimited. His offer price is determined by an increasing function of the energy generated in each time period by

$$
p=G_{h}(q), \quad h=1, \ldots, H
$$

Typically each of these functions is monotone, discontinuous and piecewise constant, or monotone continuous and piecewise linear. Along with these simple offers in each period, there are block bids defined by a set of pairs pricevolume

$$
\left(p_{n}, q_{n}\right), \quad \mathrm{n}=1, \ldots, N
$$

whose meaning is as follows: each pair represents a fixed and indivisible $q_{n}$ volume generation for each hour of a specified period under the restriction that all the volumes must be accepted or rejected together in all the periods; besides the offer is made firm only under the condition that the average price over the hours covered by the complex bid resulting in the auction process is not less than the value $p_{n}$. To limit the complexity of the notation, we have considered that the power offered by the block offers in each time period is the same; the treatment of the more general case of variable volumes is similar, with no other added difficulty than the use of a more complex notation. In addition, for the same reasons, it is assumed that the consumer demand in each hour is inelastic, being represented by the quantity $Q_{h}$ in the $h$ hour.

The allocation of generation in a way that maximizes the social welfare of the market leads to the following optimization problem: to obtain the minimum value of the function

$$
f\left(x_{1}, \ldots, x_{H} ; u_{1}, \ldots, u_{N}\right):=\sum_{h=1}^{H} \int_{0}^{x_{h}} G_{h}(x) d x+H \sum_{n=1}^{N} p_{n} q_{n} u_{n}
$$

whose first $H$ variables $x_{1}, \ldots, x_{H}$, are nonnegative and represent the continuous generation in each hour, while the remaining $N$ variables $u_{n}$ can only take values 0 or 1 , depending on whether the corresponding block is or is not included as part of the generation. Additionally, the variables are restricted by the equalities

$$
x_{h}+\sum_{n=1}^{N} q_{n} u_{n}=Q_{h}, \quad h=1, \ldots, H
$$

which represent the balance of demand and generation in each hour. Finally, by agreeing that the clearing market price at every hour is determined by the volume of the continuous generation accepted, the conditions of minimum average prices fixed in blocks orders impose the inequalities

$$
p_{n} u_{n} \leq \frac{1}{H} \sum_{h=1}^{H} G_{h}\left(x_{h}\right) \quad n=1, \ldots, N
$$

Posed in this way, the multi-period auction problem with block bids appears as a problem of mixed programming, with both continuous and integer variables, more specifically binary. In general, depending on the nature of the functions that characterize the variable generation, we have a non-linear programming problem. However, in the event that the continuous offer generation is represented by an increasing piecewise constant function, which is the case in some markets, the corresponding cost generation would be a piecewise linear function and the problem can be reformulated as mixed linear programming problem. Similarly, in the case 
of piecewise linear offer function, the problem can be formulated as a mixed quadratic problem with linear constraints. In any case, although there are software packages aimed at solving this type of programmes, when the number of variables is very high the presence of binary variables makes these problems difficult to solve. In real market operations, in order to guarantee that the scheduled transactions will be available in real time, heuristic procedures for the solution of this problem are in greater or lesser degree necessary. This is the usual mode of operation in the European markets.

\section{B. Market coupling with complex bids.}

Using notations that extend those employed in the case of a single market, the coupling of two electricity markets can be posed as the following optimization problem. The objective function is defined by

$$
f\left(\mathbf{x}_{1}, \mathbf{x}_{2} ; \mathbf{u}_{1}, \mathbf{u}_{2}\right):=f_{1}\left(\mathbf{x}_{1}, \mathbf{u}_{1}\right)+f_{2}\left(\mathbf{x}_{2}, \mathbf{u}_{2}\right)
$$

which expression includes the two real $H$-dimensional real vectors $\mathbf{x}_{1}$ and $\mathbf{x}_{2}$ representing the continuous generation volumes of each market in each hour and the $N_{i}$-dimensional binary vector $\mathbf{u}_{\mathbf{i}}$ is associated with the block order of the $i$-th market. By expressing with $Q_{1 h}$ and $Q_{2 h}$ the inelastic demands of consumers in both markets at the $h$ hour, these variables are restricted by the equalities that impose the satisfaction of the total joint demand in every hour as follows

$$
\left[x_{1 h}+\sum_{n=1}^{N_{1}} q_{1 n} u_{1 n}\right]+\left[x_{2 h}+\sum_{n=1}^{N_{2}} q_{2 n} u_{2 n}\right]=Q_{1 h}+Q_{2 h}
$$

As in the case of a single market, we have also the restrictions on the average price of each market imposed for the price lower limits of each block order, that are written as the inequalities

$$
p_{i n} u_{i n} \leq \frac{1}{H} \sum_{h=1}^{H} G_{i h}\left(x_{i h}\right) \quad 1 \leq n \leq N_{i}
$$

Notice that these restrictions affect separately each market.

Moreover, in the simplified scenario of no restrictions on the transmission network, equality of the clearing prices in both markets gives the new equations

$$
G_{1 h}\left(x_{1 h}\right)=G_{2 h}\left(x_{2 h}\right)
$$

Once more, we set aside the more general case for the sake of simplicity, but it is not difficult to extend our formulation to cover the existence of limited transmission capacity.

In principle this problem is not different in nature from the problem posed for a single market. But in this case, we have the added difficulty to perform this minimization process in a decoupled way, excluding the possibility of using the overall bids presented at the two markets, as it is considered confidential information and may not be shared between them. In the absence of complex bids, the market coupling is relatively simple, as the energy interchanged between the two markets can be obtained by a coordinator by using the net supply curves of each markets in every hour.
These curves are obtained from the functions

$$
\pi_{i h}(z):=G_{i h}\left(Q_{i h}+z\right)
$$

which represent the increases (decreases) of the prices in that market in the hour $h$ when the demands increases (decreases) in the volume $z$. If there are no transmission capacity limitations, the net energy $z_{h}$ transferred in the hour $h$ from the first to the second market is the solution of the equation

$$
\pi_{1 h}\left(z_{h}\right)=\pi_{2 h}\left(-z_{h}\right)
$$

and this solution can be easily obtained graphically from the corresponding pair of net supply curves.

When there are complex bids, it is possible to try the implementation of the previous method by imposing in each market a pre-selection of a subset of its blocks orders, the acceptance of which is assumed in each market, at the same time that the complementary set of block orders is excluded. This reduces the demand available for coupling in each market, and the corresponding pair of net supply curves can be calculated on each market .

With the net export curves associated with of the selected block bids, the market's coordinator can proceed, in the way previously described, to determine the exchanges of energy and the resulting clearing prices. Then, each market receives this information and verifies if this result is consistent with the selection of blocks proposed; otherwise both markets redefine the selection of blocks; the process is repeated until the block selections are prices-compatible.

In essence, this seems to be the method implemented in the existing European coupled markets. Obviously, the lack of clear criteria for choosing the subset of blocks makes problematic the convergence of this method. Although, at least to our knowledge, the concrete rules of these markets are not public, it is probably necessary that they use certain rules of thumb to ensure the end of the process.

\section{A dual formulation of the market coupling problem.}

In the minimization problem under which we have formulated the coupling of two markets, it is apparent that both the objective function and the restrictions are separable. To address the solution of the problem in a decentralized manner, it is quite natural then to use the Lagrangian relaxation of the restrictions that link variables of the two markets, namely those intended to the satisfaction of the overall demand and those that impose the equality of clearing prices in the two markets.

This relaxation results in a Lagrangian function which is the sum of two functions as

$$
\begin{gathered}
L_{i}\left(\mathbf{x}_{i}, \mathbf{u}_{i} ; \boldsymbol{\lambda}, \boldsymbol{\mu}\right)=f_{i}\left(\mathbf{x}_{i}, \mathbf{u}_{i}\right)+ \\
+\sum_{h=1}^{H}\left\{\lambda_{h}\left[x_{i h}+\sum_{n=1}^{N_{i}} q_{i n} u_{i n}-Q_{i h}\right]+(-1)^{i} \mu_{h} G_{i h}\left(x_{i h}\right)\right\}
\end{gathered}
$$

where we have introduced the two multiplier vectors

$$
\lambda=\left(\lambda_{1}, \ldots, \lambda_{H}\right), \quad \mu=\left(\mu_{1}, \ldots, \mu_{H}\right)
$$


Since the remaining restrictions only affects the variables of each market separately, the dual function can be constructed in decoupled way as the sum of the two functions

$$
\phi_{i}(\boldsymbol{\lambda}, \boldsymbol{\mu})=\min _{\mathbf{x}_{i}, \mathbf{u}_{i}} L_{i}\left(\mathbf{x}_{i}, \mathbf{u}_{i} ; \boldsymbol{\lambda}, \boldsymbol{\mu}\right), \quad i=1,2
$$

Notice that in the construction of each of those two functions, the only used restrictions are the inequalities in (2), which are unique to each market.

The dual function

$$
\phi(\boldsymbol{\lambda}, \boldsymbol{\mu}):=\phi_{1}(\boldsymbol{\lambda}, \boldsymbol{\mu})+\phi_{2}(\boldsymbol{\lambda}, \boldsymbol{\mu})
$$

is a concave function, typically not differentiable, one of whose sub-gradients in each point can be identified simultaneously with the determination of function values. Its maximization can be addressed iteratively using any of the various techniques based on sub-gradient techniques. Once we have obtained some optimal (or suboptimal) multipliers

$$
\left(\lambda^{*}, \mu^{*}\right) \in \arg \min \phi(\lambda, \mu)
$$

the corresponding value $\phi\left(\lambda^{*}, \mu^{*}\right)$ of dual function provides us a lower bound of the minimum value of the objective function in the primal problem. In general cases, this bound is strictly lower, because of the duality gap. Usually, the primal variable values

$$
\left(\mathbf{x}_{\mathbf{i}}^{*}, \mathbf{u}_{\mathbf{i}}^{*}\right) \in \underset{\mathbf{x}_{i}, \mathbf{u}_{i}}{\arg \min } L_{i}\left(\mathbf{x}_{i}, \mathbf{u}_{i} ; \lambda^{*}, \boldsymbol{\mu}^{*}\right)
$$

do not provide a feasible solution of the primal problem, but they can be taken as a basis to obtain feasible solutions $\left(\tilde{\mathbf{x}}_{\mathbf{i}}^{*}, \tilde{\mathbf{u}}_{\mathbf{i}}^{*}\right)$, because in most cases they are very close to the optimal solution. In this way, it is plausible that good candidates to suboptimal solutions can be obtained in a decoupled mode. In any case the sum value

$$
f_{1}\left(\tilde{\mathbf{x}}_{1}^{*}, \tilde{\mathbf{u}}_{1}^{*}\right)+f_{2}\left(\tilde{\mathbf{x}}_{2}^{*}, \tilde{\mathbf{u}}_{2}^{*}\right)
$$

gives an upper bound of the minimum value of the primal objective function. Its difference with the previously obtained lower bound can be used to estimate the quality of this suboptimal solution.

\section{NUMERICAL EXPERIENCES}

To make an initial assessment of the potentialities of the proposed algorithm, it has been applied with simulated data to solve the simplified case of the coupling of two markets with complex block bids covering two hours. In each market, it is assumed that there is a simple offer from variable generation whose price is merely proportional to the volume generation, i.e. the supply functions of continuous generation are of the form

$$
G_{i h}(q)=c_{i h} q
$$

Thus the objective function of the auction complex problem is a quadratic function, which significantly simplifies the computational burden. To model the complex bids, a number of indivisible block orders with randomly variable volumes are generated for each market. The prices of these blocks have been randomly generated around the average value of the prices assigned to these volumes by the corresponding supply functions in each market.

The algorithm has been implemented in a MATLAB simple program including the macros of the MIQP solver included in reference [8] for the solution of the mixed quadratic problems. In the numerical tests carried out the number of block orders in each market is around 20. This reduced number of blocks is used to reduce the time of calculation of the exact solution, but the results obtained seem to be independent of the number of blocks.

About in a $30 \%$ of the cases the solution obtained in the dual approach coincides with the exact solution obtained solving the auction problem joining the data from both markets. In all other cases, the set of matched block orders generated by the dual approach is almost the same as the obtained in the exact solution. This suggests the possibility of using this algorithm to obtain a good starting point for an heuristic search of better solutions, by analyzing the possibilities of including in each market some of the blocks orders paradoxically rejected.

\section{REFERENCES}

[1] ETSO. Europex "Flow-based Market Coupling". Available: http://www.etso-net.org/upload/documents/ETSO-EuroPEX_Interim report_Sept-2004-.pdf

[2] OMEL-REE-RTE-Powernext. "Definition of a new co-ordinated congestion management mechanism on the spanish-french interconnection”, Available: www.cre.fr/fr/layout/set/print/content/ download/886/13082/version/1/file/2.pdf

[3] "Trilateral Market Coupling. Algorithm Appendix", Available: www.belpex.be/uploads/media/Algorithm_Appendix_v4.6_PPOadaptations_final.pdf

[4] Meeus L "Power exchange auction platform design." $\mathrm{Ph}$. Thesis Katholieke Universiteit Leuven. Available: https://repository.libis. kuleuven.be /dspace/handle/1979/338

[5] Naranjo Salas E.J., “Análisis de la implementación del "Decentralized Market Coupling con múltiples sistemas". Tésis de Master. Universidad Pontificia de Comillas.

[6] Aguado J., Quintana V., Rosenhart W., Madrigal, M., "Co-ordinated Congestion Management of Cross-border Electricity Markets" Available: www.pscc02.org/papers/s23p03.pdf

[7] Bertsekas D.P. Convex analysis and optimization, Athena Scientific, 2003

[8] Bemporad A., Mignone D. "Hybrid Toolbox for MATLAB". Available: http://www.aut.ee.ethz.ch/ hybrid/miqp/

\section{BIOGRAPHIES}

Pedro Basagoiti obtained his Electrical Engineer degree in 1986 and his $\mathrm{PhD}$. in 1989. Since then he has been working in several companies, mainly in the IT and Energy sectors. On mars, 1998, he joined OMEL, the Spanish Electricity Market Operator, where he is the IT Systems Director.

Jose Javier Gonzalez. has obtained his Civil Engineering degree from the Madrid Polytechnic University and his M.S. from the Massachusetts Institute of Technology in 1975 and 1977 respectively. He has worked for a consulting company, and for Red Electrica de España, where he was head of the Energy Management System Department. At present, he is the market Operations Director at OMEL, the Spanish Electricity Market Operator.

Manuel Álvarez obtained the Doctor Ingeniero Industrial degree from the Universidad Politécnica de Madrid, in 1977. From this date on he has been working as an associate professor of the Departamento de Matemática Aplicada a la Ingeniería Industrial in the same University. 